\title{
Hemorragia con la administración de insulina en paciente sin antecedentes de hemorragias previas
}

\author{
Guillem Guix'1, Juan J. López-Nuñez' ${ }^{1}$ Marc Sorigué2, Cristina Tural' \\ 'Servicio de Medicina Interna, Hospital Germans Trias i Pujol, Badalona (Barcelona), España \\ ${ }^{2}$ Servicio de Hematología, Hospital Germans Trias i Pujol, Badalona (Barcelona), España
}

Recibido: 26/06/2021

Aceptado: 18/10/2021

En línea: 31/12/2021

Citar como: Guix G, López-Nuñez JL, Sorigué M, Tural C. Hemorragia con la administración de insulina en paciente sin antecedentes de hemorragias previas. Rev Esp Casos Clin Med Intern (RECCMI). 2021 (dic); 6(3): 9-11. doi: 10.32818/reccmi.a6n3a4.

Cite this as: Guix G, López-Nuñez JL, Sorigué M, Tural C. Hemorrhage with insulin administration in a patient with no history of previous haemorrhages. Rev Esp Casos Clin Med Intern (RECCMI). 2021 (Dec); 6(3): 9-11. doi: 10.32818/reccmi.a6n3a4.

Autor para correspondencia: Guillem Guix. gguix55@gmail.com

\section{Palabras clave \\ $\checkmark$ Hemorragia \\ $\checkmark$ Hemofilia \\ - Síndrome paraneoplásico \\ $\triangleright$ Diátesis \\ $\triangle$ Factor VIII}

\begin{tabular}{l} 
Keywords \\
\hline Hemorrhage \\
$\triangleright$ Hemophilia \\
$\triangleright$ Paraneoplastic syndrome \\
$\triangleright$ Diathesis \\
$\triangleright$ Factor VIII \\
\hline
\end{tabular}

\begin{abstract}
Resumen
Presentamos el caso de un varón de 69 años que, durante su estancia en Medicina Interna, presentó de forma espontánea una diátesis hemorrágica con pequeñas hemorragias en los sitios de punción de insulina. Se observó un TTPa prolongado con el posterior diagnóstico de hemofilia tipo A adquirida, con una inhibición severa del factor VIII como efecto paraneoplásico de un tumor primario de vejiga. La hemofilia adquirida es una enfermedad rara con mayor predisposición en los hombres. Suele diagnosticarse con retraso, ya que habitualmente el principal desencadenante es una hemorragia en pacientes sin antecedentes de hemorragias y con un TTPa prolongado.

Abstract
We report the case of a 69-years-old man who spontaneously presented a hemorrhagic diathesis, hematomas,
and small hemorrhages at insulin puncture sites, during his stay in the Internal Medicine department. A prolonged
activated partial thromboplastin time was observed with the following diagnosis of an acquired type A hemophi-
lia, with a severe inhibition of factor VIII as a paraneoplastic effect of a primary bladder tumor.
Acquired hemophilia is a rare disease with a predisposition for males. Usually, it is diagnosed with a certain delay
since the main trigger is a hemorrhage in patients without previous history and with a prolonged TTPa.
\end{abstract}

Puntos destacados

- La hemofilia adquirida es una entidad infrecuente con elevada mortalidad por lo que la sospecha y diagnóstico precoz son esenciales.

- En el 50\% de los casos es idiopática y en el otro 50\% se asocia a patología autoinmune, neoplásica y al embarazo mayoritariamente.

\section{Introducción}

Es conocido que el síndrome paraneoplásico puede adoptar una gran cantidad de presentaciones clínicas dependiendo de la neoplasia primaria y de otros factores, muchos de ellos desconocidos. La hemofilia adquirida es un trastorno autoinmunitario poco común que presenta una incidencia de 0,2 a 4 casos por millón de habitantes/año.

A continuación, describimos un caso ilustrativo de esta entidad que se suele diagnosticar con cierto retraso, lo que repercute en el tratamiento y pronóstico. Por este hecho, es esencial prestar atención a la presencia de hemorragias espontáneas en un paciente sin antecedentes hemorrágicos.

\section{Caso clínico}

Antecedentes y enfermedad actual

Presentamos el caso de un varón de 69 años con antecedentes de enolismo activo grave, fumador, con una fibrilación auricular anticoagulada con acenocumarol como único tratamiento. Consulta a Urgencias por clínica de infección respiratoria de vías bajas, con fiebre, tos y disnea.

\section{Exploración física y pruebas complementarias}

A la exploración inicial destaca taquipnea con crepitantes en base izquierda. Se realiza radiografía de tórax que es diagnóstica de neumonía en lóbulo inferior izquierdo complicado con derrame pleural. Asimismo, presenta antigenuria positiva para S. pneumoniae. Se realiza toracocentesis, mostrando un líquido pleural compatible con exudado no infectado.

\section{Evolución}

Se inicia tratamiento con ceftazidima, corticoides, broncodilatadores y se cursa ingreso en Medicina Interna. 
En planta, el paciente presenta repentinamente aparición de hematomas espontáneos, sobre todo en brazos, abdomen y muslos, epistaxis y hemorragias con la administración subcutánea de insulina en el tercio superior de ambos brazos. La insulina se había administrado como protocolo de hospitalización para hiperglucemia secundaria a corticoterapia.

Se realizó una analítica que mostró un tiempo de tromboplastina parcial activada prolongado que doblaba el límite alto de la normalidad y un INR de 2,7 y una anemia progresiva que había pasado de niveles de $10 \mathrm{~g} / \mathrm{dL}$ al ingreso hasta niveles de $6,5 \mathrm{~g} / \mathrm{dL}$. En ese momento se retira el tratamiento anticoagulante y se realiza una angio-TC toracoabdominal que descartó hemorragia interna, pero, al mismo tiempo, evidenció una tumoración vesical sospechosa de malignidad (Figura 1) que se confirmó también con ecografía vesical (Figura 2). Un análisis dirigido a factores de la coagulación mostró una reducción en los niveles del factor VIII y la presencia de inhibidor de FVIII.

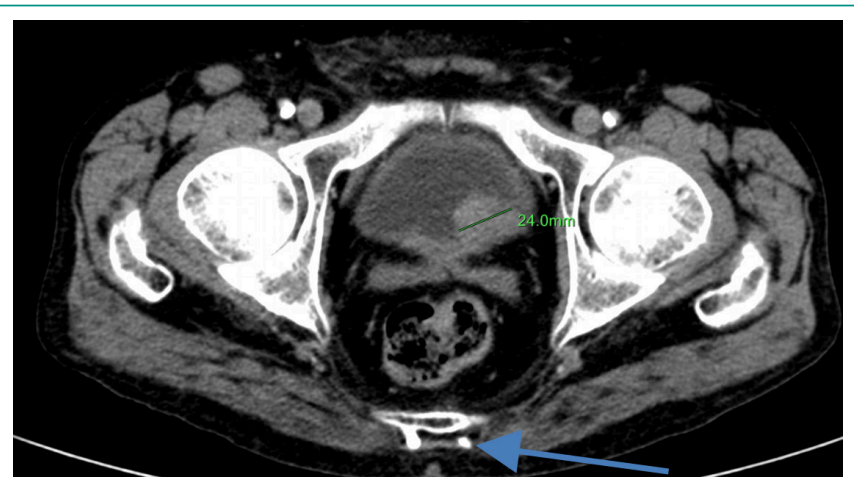

Figura 1. AngioTC que muestra tumor vesical de $24 \mathrm{~mm}$.

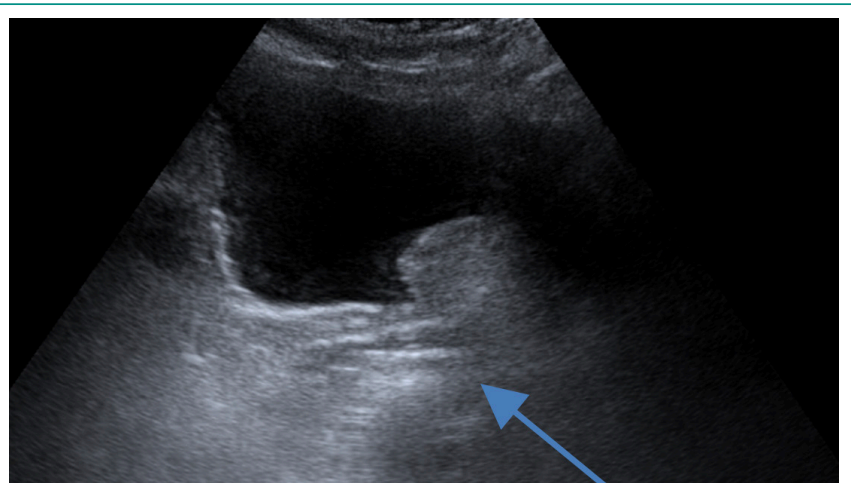

Figura 2. Misma imagen desde ecografía vesical.

Según indicaciones de hematología, se inició tratamiento con factor VII activado y bolus de 250 mg de metilprednisolona por vía intravenosa (i.v.) diarios durante 3 días, con rápida mejoría de la sintomatología hemorrágica. Además, como tratamiento inmunosupresor, se administró ciclosporina 100 mg/12 h vía oral y posteriormente metilprednisolona $30 \mathrm{mg} / 12 \mathrm{~h}$ vía i.v. recuperando los niveles normales de FVIII y alcanzando la erradicación de los inhibidores. Posteriormente se reintrodujo anticoagulación con heparina de bajo peso molecular a dosis progresivas hasta alcanzar dosis terapéuticas.

Al alta hospitalaria el paciente siguió controles por Urología. Se realizó una resección transuretral con recogida de muestra, que fue diagnóstica de carcinoma urotelial.

\section{Diagnóstico}

Hemofilia tipo A adquirida como síndrome paraneoplásico de un carcinoma urotelial de vejiga.

\section{Discusión}

La hemofilia adquirida es un trastorno autoinmune raro que presenta una incidencia de 0,2-4 casos por millón de habitantes/año y una tasa de mortalidad entre el 8-35\% según las series ${ }^{1-4}$.

Esta enfermedad muestra características particulares en comparación con la hemofilia congénita ${ }^{1-4}$. La adquirida presenta como puntos de sangrado preferentes: los tejidos blandos, el músculo o el tracto gastrointestinal; mientras que la hemartrosis es el punto típico en la congénita. Suele tener una presentación aguda en un paciente sin historia previa de sangrado, con mayor incidencia en varones y un patrón de edad bifásica: adultos mayores y mujeres embarazadas.

Es una enfermedad con un habitual retraso diagnóstico, ya que su clínica más frecuente es un sangrado espontáneo inespecífico en más del $80 \%$ de los casos $^{2}$.

En la literatura, un tiempo parcial de tromboplastina activada (TTPA) prolongado que no se corrige con el «mixing test», niveles plasmáticos reducidos de FVIII y la presencia de un inhibidor contra el FVIII son factores cruciales para sospechar una de las siguientes cuatro patologías ${ }^{1-3,5}$ : una deficiencia de factor, una hemofilia adquirida, una coagulación intravascular diseminada (CID) o presencia de anticoagulante lúpico. Al realizar el «mixing test», que mezcla plasma del paciente con plasma de un paciente normal, si el TTPA mejora, sugiere una deficiencia de factor; por el contrario, si no hay mejoría de los niveles de TTPA, tenemos que centrarnos en una de las otras tres patologías.

En cuanto a la etiología en nuestro paciente, en el momento de aparición de las hemorragias, había mejorado de la neumonía inicial y no presentaba signos de sepsis como para atribuirlo a una CID. Además, a posteriori, se le diagnosticó un carcinoma urotelial.

Según la literatura, aproximadamente el 50\% de los casos son idiopáticos². El otro 50\% se divide en enfermedad maligna subyacente (10-15\%), sobre todo tumor sólido (los principales son próstata y pulmón) ${ }^{4}$, linfoma y leucemia linfocítica crónica. A continuación, se encuentran las enfermedades autoinmunes (10-11\%), destacando la artritis reumatoide como la principal; el embarazo (8-9\%) y otras causas menos frecuentes (infecciones, inducidas por fármacos, enfermedades dermatológicas).

En cuanto al tratamiento, existe poca evidencia debido a la falta de ensayos clínicos prospectivos que comparan las diferentes estrategias. Las guías se basan en estudios retrospectivos, recomendaciones de expertos y registros europeos y estadounidenses. Debemos diferenciar por un lado el tratamiento de la hemorragia aguda donde, en la actualidad, la primera línea consiste en los denominados agentes bypass, como el FVII recombinante activado (rFVIla). Se recomienda administrar 90-120 $\mathrm{mgs} / \mathrm{kg}$ cada 2-3 h y aumentar progresivamente los intervalos según la progresión de complejo de protrombina activada $(\mathrm{CPA})^{2,3}$ hasta dosis convencionales de 50-100 $\mathrm{\mu gs} / \mathrm{kg}$ cada 8-12 h sin superar los $200 \mu \mathrm{gs} / \mathrm{kg} / \mathrm{día}$.

Otras opciones serían el FVIII porcino recombinante y la desmopresina 3, pero estas dos han mostrado una peor tasa de control de la hemorragia en algunas series con una diferencia estadísticamente significativa ${ }^{4}$.

Por otro lado, en relación con la terapia inmunosupresora, el tratamiento más estudiado es la combinación de corticoides i.v (1 mg/kg/día) asociados a ciclofosfamida i.v (1-2 mg/kg/día) entre 3 y 5 semanas $\mathrm{s}^{3,4,6}$. También se ha utilizado el rituximab de segunda línea, pero con peores resultados y se han estudiado otras alternativas como Emicizumab?

Finalmente, hay que mencionar que uno de los principales efectos adversos de los agentes bypass son los eventos trombóticos. En el caso presentado, se 
observó una trombosis de la vena cava durante el seguimiento que obligó a interrumpir el tratamiento con rFVIla. En la literatura, el registro EACH2 describe un 2,9\% de eventos tromboembólicos entre los pacientes tratados con rFVIIa 2,8 .

\section{Conclusiones}

La hemofilia adquirida es una enfermedad rara con mayor incidencia en varones (especialmente si se excluyen las mujeres embarazadas). Suele diagnosticarse con cierto retraso ya que habitualmente el principal síntoma es una hemorragia en pacientes sin antecedentes y con un TTPa prolongado El retraso en el diagnóstico también enlentece el inicio de la terapia hemostática pero no afecta a su duración ni a los niveles iniciales de inhibidores del FVIII.

La etiología principal es idiopática, pero en los casos de etiología conocida las más frecuentes son las neoplasias sólidas (próstata y pulmón), seguidas de las enfermedades autoinmunes. El patrón de hemorragias es diferente al de la congénita y el principal tratamiento son los agentes bypass. El tratamiento inmunosupresor más utilizado es la corticoterapia combinada con ciclofosfamida.

\section{Bibliografía}

1. Kessler CM, Knöbl P. Acquired haemophilia: an overview for clinical practice. Eur J Haematol. 2015; 95 Suppl 81: 36-44. doi: 10.1111/ejh.12689.
2. Knoebl P, Marco P, Baudo F, Collins P, Huth-Kühne A, Nemes L et al. Demographic and clinical data in acquired hemophilia A: results from the European Acquired Haemophilia Registry (EACH2). J Thromb Haemost. 2012; 10(4): 622-31. doi: 10.1111/j.1538-7836.2012.04654.x.

3. Mingot-Castellano ME, Núñez R, Rodríguez-Martorell FJ. Acquired haemophilia: Epidemiology, clinical presentation, diagnosis and treatment. Med Clin (Barc). 2017; 148(7): 314-322. doi: 10.1016/j.medcli.2016.11.030.

4. Napolitano M, Siragusa S, Mancuso S, Kessler CM. Acquired haemophilia in cancer: A systematic and critical literature review. Haemophilia. 2018; 24(1): 43-56. doi: 10.1111/hae.13355.

5. Wan Ab Rahman WS, Abdullah WZ, Husin A, Nik Mohd Hassan NFF, Hassan MN, Zulkafli Z. Epidemiology, spectrum of clinical manifestations and diagnostic issue of acquired haemophilia: A case series. Malays J Pathol. 2019; 41(2): 185-189.

6. Möhnle P, Pekrul I, Spannagl M, Sturm A, Singh D, Dechant C. Emicizumab in the Treatment of Acquired Haemophilia: A Case Report. Transfus Med Hemother. 2019; 46(2): 121-123. doi: 10.1159/000497287.

7. Delgado J, Jimenez-Yuste V, Hernandez-Navarro F, Villar A. Acquired haemophilia: review and meta-analysis focused on therapy and prognostic factors. Br J Haematol. 2003; 121 (1): 21-35. doi: 10.1046/j.1365-2141.2003.04162.x.

8. Collins PW, Chalmers E, Hart D, Jennings I, Liesner R, Rangarajan S, Talks K, Williams M, R M Hay C; United Kingdom Haemophilia Centre Doctors' Organization. Diagnosis and management of acquired coagulation inhibitors: a guideline from UKHCDO. Br J Haematol. 2013; 162(6): 758-73. doi: 10.1111/ bjh.12463. 